\title{
Estudio comparativo mediante Microscopía Electrónica de dos tipos de ionómeros de vidrio sometidos a un proceso químico
}

\author{
Comparative study by electron microscopy of two types of glass-ionomers undergoing a chemical \\ process
}

Raisa Severino ${ }^{1}$, Fanny Lazo $^{2}$, Ruperto Severino $^{3}$, Dan Vivas-Ruiz ${ }^{2}$, Gustavo A. Sandoval ${ }^{2}$

\section{RESUMEN}

Objetivo: Analizar mediante microscopía electrónica de barrido dos tipos de ionómeros de vidrio sometidos a un proceso químico y realizar la comparación respectiva. Materiales y Métodos: Se utilizaron discos de dos tipos de cemento de ionómero de vidrio, uno convencional y el otro modificado con resina. Ambos ionómeros fueron sometidos a un proceso químico de solubilidad durante 30 días siguiendo el protocolo establecido por la ISO 4049. Se realizó la microscopía electrónica al inicio y final del período de tiempo, analizando la morfología y características de estos ionómeros y realizando las comparaciones respectivas. Resultados: Las microfotografías de la microscopía electrónica de barrido muestran cambios significativos en las superficies de los discos luego de haber sido sometidos al proceso químico durante 30 días, las microfotografías muestran que el ionómero de vidrio convencional fue el que mostró mayor cantidad de gaps y a la vez con mayor profundidad, mayores fisuras e irregularidades en su superficie. Conclusión: La microscopía electrónica de barrido evidenció cambios en la superficie de los discos de ionómero de vidrio, siendo el ionómero de vidrio convencional el que evidenció mayores cambios en su estructura.

Palabras clave: microscopía electrónica, ionómero de vidrio, solubilidad.

\begin{abstract}
Objective: To analyze by scanning electron microscopy two types of glass-ionomer subjected to a chemical process and make the respective comparison. Materials and Methods: Discs of two types of glass-ionomer cement were used, a conventional one and the other modified with resin, both ionomers were subjected to a chemical process of solubility for 30 days following the protocol established by the ISO 4049. Electron microscopy al baseline and in the end of the period of time was performed by analyzing the morphology and characteristics of these ionomers and performing the respective comparisons. Results: The microphotographs of the scanning electron microscopy show significant changes in the disc surfaces after being subjected to the chemical process for 30 days, and was the conventional ionomer which showed more gaps and deeper, greater cracks and irregularities on the surface. Conclusion: The electron microscopy showed changes on the surface of the glass-ionomer discs, being the conventional one which showed major changes in its structure.
\end{abstract}

Key words: electron microscopy, glass-ionomer, solubility.

\section{INTRODUCCIÓN}

La cementación dental es un procedimiento habitual en la restauración de piezas dentarias con coronas destruidas o con caries amplias. Las restauraciones indirectas (inlay y onlay) que involucran la incorporación de estructuras metálicas, cerámicas o de resina, requieren del uso de agentes que compensan la interfase diente-restauración denominados comúnmente

\footnotetext{
${ }^{1}$ Cirujana Dentista. Facultad de Odontología. Universidad Nacional Mayor de San Marcos. Lima 01. Perú

${ }^{2}$ Laboratorio de Biología Molecular. Facultad de Ciencias Biológicas. Universidad Nacional Mayor de San Marcos. Lima 01. Perú

${ }^{3}$ Laboratorio de Zoología de Invertebrados Acelomados y Pseudocelomados. Facultad de Ciencias Biológicas.

Universidad Nacional Mayor de San Marcos. Lima 01. Perú
} 
como agentes cementantes cuya función primordial es permitir la retención de la restauración por el aumento del área de contacto entre el diente y ella, además de mejorar el sellado marginal. [1]

La selección del agente cementante es una decisión de suma importancia en una secuencia de pasos para determinar el éxito a largo plazo de tratamiento dental; debido a que, al ser expuestos al medio bucal, los agentes cementantes experimentan diversos procesos que conllevan a su degradación afectando de esta manera su calidad y estabilidad. Por tal razón, un buen agente cementante debe reunir una serie de requisitos como fluidez, adecuado grosor, capacidad de retención, un buen aislamiento térmico y sobre todo una baja solubilidad parámetro relacionado tanto con la tasa de degradación como con la biocompatibilidad entre material y la estructura dentaria. [2]

Dentro de los agentes cementantes, el ionómero de vidrio se posiciona como uno de los materiales más empleados por los odontólogos por su excelente adhesividad, alta estética y su liberación de flúor que contribuye a prevenir futuras caries. Desde su aparición, el ionómero de vidrio ha sido perfeccionado con el avance de la tecnología, es así que ahora se cuenta con ionómeros de vidrio modificado con resina que revoluciona la estructura de este agente cementante $y$ exhibe nuevas propiedades físico-químicas ventajosas que se traducen en éxito significativo de los tratamientos dentales. $[1,3]$

En el presente trabajo se realiza un análisis comparativo, mediante microscopía electrónica de barrido (SEM), de la estructura de ionómeros de vidrio sometidos a un proceso químico con el objetivo de resaltar las diferencias estructurales y la implicancia que esto puede tener en los tratamientos.

\section{MATERIALES Y METODOS}

\section{Elaboración de los discos}

Se fabricaron siguiendo las indicaciones de la ISO 4049, utilizando un molde metálico de $15,0 \pm 0,1 \mathrm{~mm}$ de diámetro y $1,0 \pm 0,1 \mathrm{~mm}$ de profundidad. Las mezclas fueron inyectadas en el molde mediante el sistema Centrix. Las indicaciones hechas por el fabricante fueron seguidas, tanto para el cemento ionómero de vidrio (IVC) GC Gold Label y para el ionómero modificado con resina (IVMR) GC Fuji Plus. Una vez obtenidos los discos, estos fueron pulidos con disco de carburo y motor de baja velocidad para retirar los excedentes. Posteriormente se midieron con ayuda de un vernier para verificar las dimensiones de ellos. Se utilizaron 24 discos con IVC y 24 con IVMR, seleccionados de manera aleatoria.

\section{Prueba de solubilidad}

Los discos fueron llevados a una estufa a 37 ${ }^{0} \mathrm{C}$ y luego pesados en una balanza analítica hasta obtener una masa constante. Luego fueron colocados en frascos rotulados que contenían $10 \mathrm{~mL}$ de agua destilada y mantenidos a $37{ }^{\circ} \mathrm{C}$, en la que permanecieron 30 días, para luego ser retirados de la estufa, lavados con agua destilada, secados hasta quedar libres de humedad visible. Posteriormente los discos fueron colocados nuevamente a $37{ }^{\circ} \mathrm{C}$ hasta obtener una masa constante. Para obtener los datos de volumen, y solubilidad se utilizó las fórmulas matemáticas dadas por la ISO 4049.

\section{Preparación de los discos para SEM}

Se elaboraron en serie, siguiendo las indicaciones del fabricante y el protocolo establecido por la ISO 4049 [4] para la conformación de los discos. De las muestras confeccionadas se seleccionó al azar un grupo de ellas tanto del IVC como del IVMR para ser sometidas a microscopia electrónica de barrido y así poder tener las microfotografías iniciales, y el resto preparadas para ser sometida al proceso de sorción y solubilidad de acuerdo a la norma ISO 4049, por un período de 30 días a $37{ }^{\circ} \mathrm{C}$, posterior a ello se procedió a la preparación de las muestras para su estudio SEM.

Para poder pasar por la evaluación de microscopía las muestras tuvieron que ser sometidas a un proceso de galvanización con oro de $24 \mathrm{k}$ a través de un equipo especializado (SPI MODULETM Sputter/Carbon Coater) por un tiempo promedio de 5 minutos.

\section{Visualización por SEM}

Las muestras metalizadas fueron introducidas al Microscopio electrónico de Barrido (FEI-Inspect S50) en modo de alto vacío y con $12.5 \mathrm{kV}$. Las microfotografías se tomaron en un rango de aceleración de voltaje de 2,00-3,00 Kv, a un mismo aumento de (1000 X). Los archivos fueron procesados por medio del ordenador acoplado al microscopio. 


\section{RESULTADOS}

Los datos recolectados fueron procesados por el paquete estadístico SPSS 21,0 para Windows. Se realizó el test de Krustal Wallis donde se obtuvo $\mathrm{p}=0,000$ ( $\mathrm{p}<0,05)$, lo que nos indicó que si existían diferencias significativas para solubilidad entre estos dos cementos. La prueba de T-Student fue usada para comparar las medias en casos que las muestras cumplían con la normalidad y homogeneidad de varianzas y en los otros casos se utilizó la prueba de U. de Mann-Whitney. Los resultados muestran que para el proceso de solubilidad el IVC mostró valores cercanos a $36,42 \pm 14,59$ y el IVMR de $-4,52 \pm 2,38$ a los 30 días, no mostrando signos de solubilidad el IVMR en el mismo período de tiempo (Tabla $1)$.

Con el efecto de evaluar el efecto de la solubilidad sobre el cemento ionómero de vidrio convencional y modificado con resina por microscopía electrónica, se tomaron microfotografías, lo cual permitió la observación y caracterización superficial de las muestras tanto iniciales como las que pasaron 30 días inmersas en agua.

Los resultados muestran que en el estado inicial el ionómero de vidrio convencional
(Fig. 1A) mostró presencia de detritus, fisuras con relleno del material y distantes entre sí, así como micro gaps con bordes irregulares. Mientras que en el ionómero de vidrio modificado con resina (Fig. 1B) presentó una superficie menos irregular con relleno del material sobre la superficie, con escasas fisuras, y micro-gaps superficiales, escasos y distantes.

Las microfotografías luego que las muestras hayan pasado el proceso de solubilidad evidencian que el ionómero de vidrio convencional (Fig. 1C) mostró estrías en la superficie, fisuras más marcadas, lineales y profundas, restos de material dispuestos en la superficie y en las fisuras, y estas alargadas y uniéndose entre sí por micro-fisuras, gaps con mayor diámetro y profundidad, rodeados de una malla de fisuras y con restos de material dentro de los gaps. Por otro lado el ionómero de vidrio modificado con resina (Fig. 1D) mostró algunas zonas con restos de material, fisuras irregulares, finas y no tan extensas, gaps con bordes irregulares, de menor diámetro y profundidad y rellenos de restos del material.

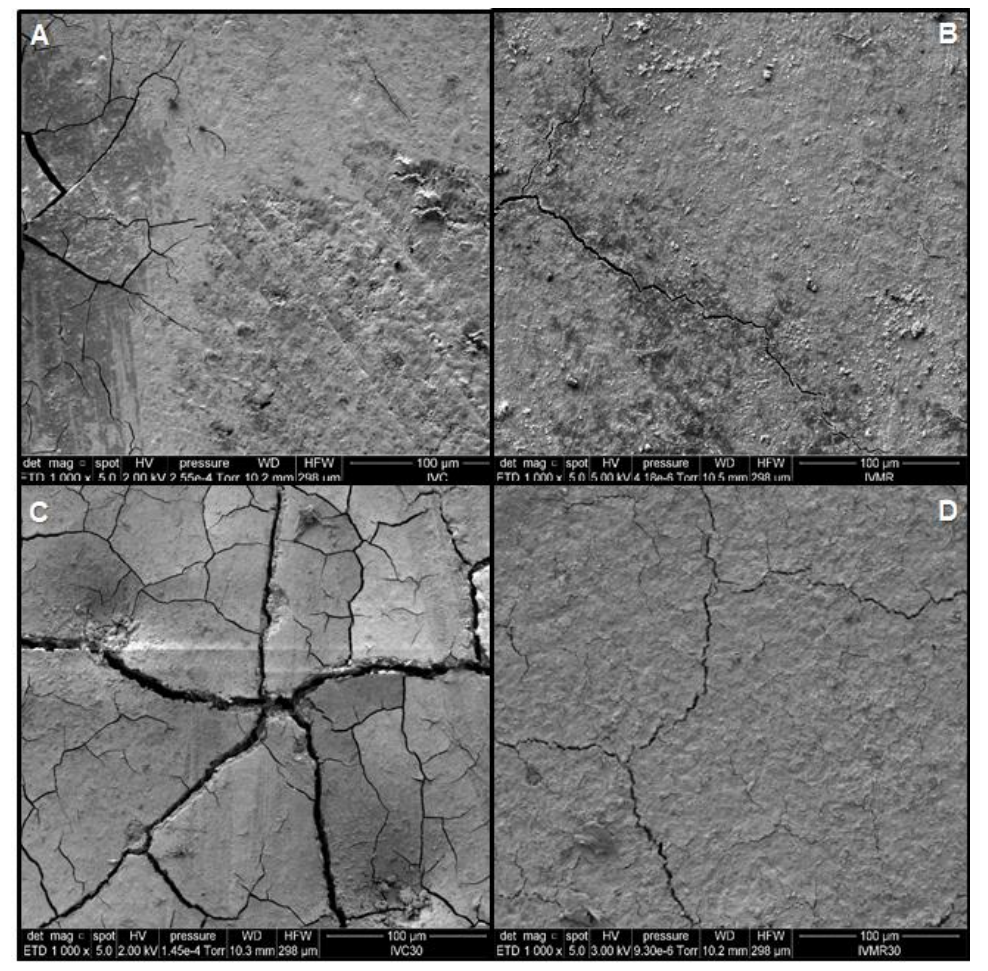

Fig. 1. Microfotografías: A) Cemento Ionómero de Vidrio Convencional antes de ser sometido al proceso de solubilidad. B) Cemento Ionómero de Vidrio Modificado con Resina antes de ser sometido al proceso de solubilidad. C) Cemento Ionómero de Vidrio Convencional luego de ser sometido al proceso de solubilidad durante 30 días. D) Cemento Ionómero de Vidrio Modificado con Resina luego de ser sometido al proceso de solubilidad durante 30 días. 
Tabla 1. Resultados de la prueba de solubilidad

\begin{tabular}{ccccc}
\hline Prueba & $\begin{array}{c}\text { Tipo de } \\
\text { ionómero }\end{array}$ & $\begin{array}{c}\text { Peso mínimo } \\
\text { del disco }\end{array}$ & $\begin{array}{c}\text { Peso máximo } \\
\text { del disco }\end{array}$ & Promedio \\
\hline Solubilidad & IVC & 23,76 & 67,34 & $36,42 \pm 14,59$ \\
\cline { 2 - 5 } & IVMR & $-9,05$ & $-2,26$ & $-4,52 \pm 2,38$ \\
\hline
\end{tabular}

\section{DISCUSIÓN}

Estos materiales una vez expuestos a medio oral sufren diversos procesos, uno de ellos la solubilidad, ya sea en mayor o menor cantidad, que conlleva a una degradación de los mismos, afectando la calidad y longevidad de nuestros tratamientos [5].

Diversos autores han destacado la vital importancia de estudiar estos procesos pues alteran la estructura de los materiales, afectando sus propiedades mecánicas, ópticas y la biocompatibilidad [6-10].

Los resultados de la presente investigación ponen en manifiesto las diferencias entre estos dos materiales a un nivel microscópico observando lo que no podemos identificar a simple vista y es clave para el éxito del tratamiento.

Keyf et al. [10] determinaron que la superficie del ionómero de vidrio convencional luego de ser inmerso en agua presentó cambios estructurales y morfológicos en la superficie coincidiendo con lo hallado en esta investigación. Adicionalmente las microfotografías evidenciaron clara diferencia entre estos dos tipos de ionómeros, por un lado el cemento ionómero de vidrio convencional inicialmente presentaba una superficie parcialmente lisa, con pequeños micro gaps y fisuras leves, sin embargo luego de 30 días y haber pasado el proceso de solubilidad, la superficie es otra, estos cambios vistos in vitro ocurren de la misma manera y según lo reportado en estudios hechos por otros autores $[6,7,9]$ estos cambios ocurren a mayor velocidad en la cavidad bucal, lo que conlleva a un desgaste del cemento imperceptible a simple vista pero que va afectando la calidad del tratamiento.

Por otro lado, las microfotografías del cemento ionómero de vidrio modificado con resina nos muestran una superficie mucho más lisa, con gaps escasos y finas fisuras, sin embargo luego de los 30 días inmersos en gua las fisuras solo llegaron a profundizarse al igual que los gaps, pero a diferencia del ionómero de vidrio convencional, estas fisuras y gaps estaban rellenos de restos del material en su interior, teniendo así una clara evidencia de que este cemento presentó una expansión higroscópica, lo que se traduce como una expansión del material, confiriéndole a este un mejor sellado con la estructura dentaria, así como con el material restaurador, por consiguiente obteniendo una mayor calidad del tratamiento que realizamos $[12,13]$.

\section{CONCLUSIONES}

Luego de 30 días el cemento ionómero de vidrio modificado con resina empezó a retener líquido en su estructura originando una expansión higroscópica. La microscopía electrónica de barrido evidenció la diferencia entre estos dos cementos a un nivel de cambios imperceptibles a simple vista, cambios significativos en la superficie de estos cementos luego de 30 días inmersos en agua, todo esto repercutiendo en la calidad y longevidad de los tratamientos.

\section{AUTOR DE CORRESPONDENCIA}

C.D. Raisa Severino Lazo.

Facultad de Odontología.

Universidad Nacional Mayor de San Marcos

Av. Amezaga s/n-Ciudad Universitaria, Lima - Perú

E-mail: raisa.severinol@gmail.com

\section{REFERENCIAS BIBLIOGRÁFICAS}

1. Carlos Carrillo Sánchez. Actualización sobre los cementos de ionómero de vidrio, 30 años (1969-1999). Rev. ADM. 2000 Marzo-Abril; Vol. 57(2):65-71p.

2. Nocchi Conceição, Ewerton. Odontología Restauradora: Salud y Estética. 2da Edición. Buenos Aires: Editorial Médica Panamericana; 2008.187-202p. 
3. Kenneth W. Aschheim. Esthetic dentistry: A clinical Approach to techniques and materials.3ra Edición. St. Louis, Missouri: Editorial Elsevier Mosby; 2015. 55-280p.

4. International Standard ISO 4049: Dentistry-Polymer-based restorative materials. Fourth Edition; 2009.

5. Lad P. P., Kamath M., Tarale K., Kusugal P. B. Practical clinical considerations of luting cements: A review. Journal of International Oral Health: JIOH. 2014; Vol. 6(1):116-120p

6. M. A. Cattani-Lorente, V. Dupuis, F. Moya, J. Payan, J-M. Meyer. Effect of water on the physical properties of resinmodified glass ionomer cements. Academy of Dental Materials. 1999; Vol. 15:71-78p.

7. Vaca M. J., Ceballos L., Fuentes M. Y., Osorio R., Toledano M., García-Godoy F. Sorción y solubilidad de materiales formulados con resina. Avances en Odontoestomatología. 2003; Vol. 19(6):283-289p.

8. Beriat, Nilufer, Tulunoglu Ibrahim. Evaluation of the Solubility of resinmodified glass ionomer cements. Rev. Fac. Odontología Univ. Hacettepe. 2007; Vol. 30(3):3-7p

9. H. Hajmiragha, S. Nokar, M. Alikhasi, S. Nikzad, H. Dorriz. Solubility of three luting cements in dynamic artificial saliva. Journal of Dentistry of Tehran University of Medical Sciences. 2008; Vol. 5(3):9598p.

10.Filiz Keyf, S. Hakan Tuna, Murat Sen, Agnes Safrany. Water sorption and solubility of different lutting and restorative dental cements. Turk J. Med Sci. 2007; Vol. 37(1):47-55p.

11.Barrancos, Mooney Julio. Operatoria dental: integración clínica. 4ta Edición. Patricio Barrancos. Buenos Aires: Editorial Médica Panamericana; 2006. 755-770p.

12.L. A. Knobloch, R. E. Kerby, K. McMillen, N. Clelland. Solubility and sorption of resin-based luting cements. Academy of Operative Dentistry. 2000 SeptiembreOctubre. Vol. 25(5):434-440p.

13.José Carlos Rabelo Ribeiro; Ueide Fernando Fontana; Marcos Ribeiro Moyses; Andréa Candido dos Reis; Sérgio Candido Dias; João Gustavo Rabelo Ribeiro. Evaluación de la solubilidad y desintegración de cementos de ionómero de vidrio modificados con resina y compómeros en función de protección superficial. Revista de Odontología da UNESP. 2006; Vol. 35(4):247-252p.

14.Bashir M. Saad, Toros Alcan. In vivo disintegration of luting cements. OHDMBSC. 2005 Setiembre. Vol. 4(3):34-44p.

15.Beriat, Nilufer, Tulunoglu Ibrahim. Evaluation of the Solubility of resinmodified glass ionomer cements. Rev. Fac. Odontología Univ. Hacettepe. 2007; Vol. 30(3):3-7p.

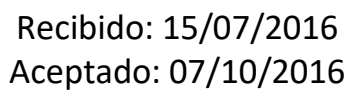

Recibido: $15 / 07 / 2016$ Aceptado: 07/10/2016 